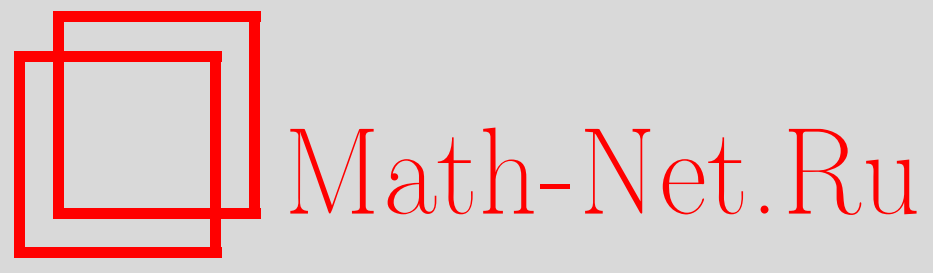

В. Б. Пеньков, Л. В. Левина, Е. А. Новиков, Строгое решение задачи о состоянии линейно-упругого изотропного тела под воздействием полиномиальных объемных сил, Вестн. Сам. гос. техн. ун-та. Сер. Физ.-мат. науки, 2021, номер 3, 475-490

DOI: https://doi.org/10.14498/vsgtu1864

Использование Общероссийского математического портала MathNet.Ru подразумевает, что вы прочитали и согласны с пользовательским соглашением

http: //www . mathnet.ru/rus/agreement

Параметры загрузки:

IP : 54.89 .56 .158

26 апреля 2023 г., 17:54:29

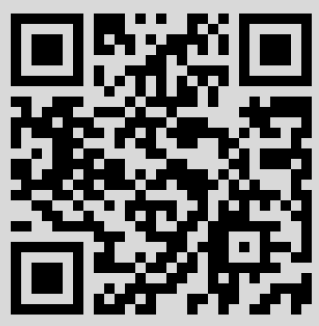


УДК 539.3

\title{
Строгое решение задачи о состоянии линейно-упругого изотропного тела под воздействием полиномиальных объемных сил
}

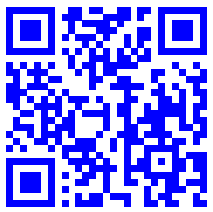

\author{
(С) В. Б. Пенъков, Л. В. Левина, Е. А. Новиков
}

Липецкий государственный технический университет, Россия, 398055, Липецк, ул. Московская, 30.

\section{Аннотация}

При решении краевых задач о построении напряженно-деформированного состояния линейно-упругого изотропного тела важным шагом является отыскание внутреннего состояния, порожденного силами, распределенными по занятой телом области. В классическом варианте существует численный способ оценки состояния в любой точке тела, базирующийся на сингулярно-интегральном представлении Чезаро. В варианте консервативных объемных сил возможно выписывание решений в аналитической форме. При произвольных регулярных воздействиях механической и иной физической природы силы потенциальными не являются и подходы Папковича-Нейбера и Аржаных-Слободянского оказываются бессильными. Кроме этого, решение нелинейных задач эластостатики средствами метода возмущений, а также использование при решении задач для исследования многополостных тел алгоритма Шварца приводят к необходимости решения последовательности линейных задач. При этом в обязательном порядке зарождаются фиктивные объемные силы, имеющие, как правило, полиномиальный характер.

Разработанный авторами ранее метод оценки напряженно-деформированного состояния тела, вызванного воздействием полиномиальных объемных сил, представляемых в декартовых координатах, получил развитие. Внутреннее состояние восстанавливается в строгом соответствии с силами, статически воздействующими на односвязное ограниченное линейно-упругое тело. Предложены и описаны эффективный метод построения решения и алгоритм его компьютерной реализации. Продемонстрированы тестовые расчеты. Выполнен анализ состояния шара, находящегося под воздействием суперпозиции объемных сил различного

\section{Научная статья}

( )(7) Контент публикуется на условиях лицензии Creative Commons Attribution 4.0 International (https://creativecommons.org/licenses/by/4.0/deed.ru)

\section{Образец для цитирования}

Пеньков В. Б., Левина Л. В., Нов иков Е. А. Строгое решение задачи о состоянии линейно-упругого изотропного тела под воздействием полиномиальных объемных сил // Вестн. Сам. гос. техн. ун-та. Сер. Физ.-мат. науки, 2021. Т. 25, № 3. С. 475-490. https://doi.org/10.14498/vsgtu1864.

\section{Сведения об авторах}

Виктор Борисович Пеньков (10) https://orcid.org/0000-0002-6059-1856 доктор физико-математических наук; профессор; каф. общей механики; e-mail: vbpenkov@mail.ru

Любовъ Владимировна Левина (D) https://orcid.org/0000-0002-7441-835X кандидат физико-математических наук; доцент; каф. прикладной математики; e-mail: satalkina_lyubov@mail.ru

Евгений Александрович Новиков (1D https://orcid.org/0000-0001-5606-5207 аспирант; кафедра общей механики; e-mail: 89513027802@mail.ru 
характера при различных соотношениях параметров, подчеркивающих уровень влияния этих факторов. Результаты оформлены графически. Сделаны следующие выводы:

а) обоснована процедура выписывания напряженно-деформированного состояния от объемных сил, представляемых полиномами от декартовых координат;

б) алгоритм реализован в вычислительной системе Mathematica и проведено тестирование на многочленах высокого порядка;

в) проведен анализ квазистатического состояния линейно-упругого изотропного шара, подверженного воздействию сил гравитации и инерции при различных сочетаниях параметров, отвечающих вариантам медленного, быстрого, компенсационного (инерционные силы соразмерны с гравитационными) вращений.

Отмечены перспективы развития нового подхода на класс ограниченных и неограниченных тел, содержащих произвольное число полостей.

Ключевые слова: объемные силы, частное решение, напряженно-деформированное состояние от объемных сил, линейная эластостатика, теория упругости, частное решение уравнений Ламе.

Получение: 22 апреля 2021 г. / Исправление: 7 сентября 2021 г. / Принятие: 20 сентября 2021 г. / Публикация онлайн: 30 сентября 2021 г.

Введение. Восстановление полей напряженно-деформированного состояния эластостатических тел, обусловленных объемными силами, относится к теме построения частного решения задач математической физики для объектов, описываемых системой соотношений, определяющих упругую среду:

1) соотношений Коши, выражающих тензор деформаций через вектор перемещений точек;

2) обобщенного закона Гука, связывающего между собой компоненты тензоров напряжений и деформаций;

3) уравнений равновесия, в которых объемные силы согласуются с полем тензора напряжений.

Последние уравнения как раз и характеризуют систему уравнений как неоднородную и требуют при построении решения краевой задачи предварительно восстанавливать в форме частного решения внутреннее состояние, отвечающее объемному силовому воздействию.

Классические положения о возможностях реализации такой процедуры известны [1-6]. В общем случае за частное решение отвечает интегральное представление Чезаро [1-3], выражающее поле вектора перемещения через сингулярный интеграл пространственного типа, но не позволяющее получить для тел произвольной формы даже приближенное аналитическое выражение. Использование точечного численного подхода при применении способов в построении численно-аналитических решений, форма представления которых имеет вид аналитических выражений, весьма неудобно.

Приведение совокупности определяющих соотношений к системе трех дифференциальных уравнений (каждое второго порядка) относительно трех дважды дифференцируемых функций, описывающих перемещение точек тела в форме Ламе $[2,3]$, позволило П. Ф. Папковичу и Г. Ф. Нейберу [7] выписать общее решение, но с существенным ограничением на класс допускаемых к рассмотрению объемных сил - они должны быть потенциальными. Эффек- 
тивному развитию такого подхода служат общие решения И. С. Аржаных, М. Г. Слободянского [2, 3], записанные как для односвязного ограниченного тела, так и для внешности ограниченной полости. Их суперпозиция позволяет строить решения для произвольных многополостных тел.

Отметим, что традиционные силы, порожденные причинами чисто механического характера (силы тяжести, силы инерции при равномерном вращении тел, силы гравитационного взаимодействия), обладают именно такими свойствами [8-11]. В современных условиях развития науки и техники возникли новые причины зарождения сил, распределенных по области, занятой упругим телом. Обнаружены причины нарушения межмолекулярного взаимодействия на наноуровне [12]. Обеспечение специфически направленных потоков заряженных частиц сквозь упругое тело, помещенное во внешнее магнитное поле, порождает силы электромагнитного взаимодействия Лоренца [13]. Эти силы уже не относятся к классу консервативных, но на упругое состояние тела оказывают прямое воздействие.

Еще одна причина, порождающая формальные объемные силы, связана со средствами решения неоднородных и термоупругих [14], геометрически [15] и физически [16,17] нелинейных задач средствами метода возмущений. Здесь решение нелинейной проблемы сводится к последовательности шагов, на каждом из которых решается задача линейного типа для соотношений классического характера, но обязательно содержащая фиктивную объемную силу, как правило, полиномиального характера [14].

Способ определения частного решения для такого случая построен - доказана теорема о существовании базиса пространства полиномиальных объемных сил, указаны эффективные алгоритмы выписывания частного решения [18-20]. Приведены примеры их эффективного применения. Эти алгоритмы связаны с необходимостью вычисления скалярных произведений элементов базиса, что при высоких размерностях полиномов, описывающих внутреннее состояние, требует существенных затрат времени счета.

Разработка более эффективного способа выписывания внутреннего состояния, порождаемого полиномиальными объемными силами, является темой актуальной, ресурсосберегающей.

Цель соответствующего исследования заключается в создании метода, позволяющего выписывать напряженно-деформированное состояние от полиномиальных объемных сил, минуя необходимость вычисления кратных интегралов при решении задачи. Эта цель достигнута, о чем свидетельствует изложенное ниже.

1. Полиномиальные базисы объемных сил. Полиномиальным называем континуальное пространство $X_{p}$ объемных сил, описываемое многочленами. Это определение характеризует его как линейное. Будем полагать тело односвязным, ограниченным, занимающим область $V$ с границей $\partial V$. Это пространство имеет сепарабельный базис [19], полнота которого и линейная независимость его элементов строго доказаны [20]. Описаны два способа формирования отрезков базиса $X_{k}$ произвольного порядка $k$ :

1) обеспечивающий алгоритм, одновременно служащий конструктивным доказательством упомянутых обязательных свойств элементов базиса;

2) сортировочный алгоритм, ведущий к достижению цели ресурсосберегающими средствами. 
Реально построенный любым способом исходный базис $X_{k}$ будем называть опорным базисом.

В любом варианте организации опорного базиса исходим из того, что произвольный моном $w=x^{\alpha} y^{\beta} z^{\gamma},\{x, y, z\} \in \mathbb{R}^{3}$ порождает в соответствии с определяющими соотношениями линейной однородной эластостатической среды (изотропной, анизотропной) три варианта полиномиальных сил в соответствии с цепочкой действий

$$
\mathbf{u} \rightarrow \hat{\boldsymbol{\varepsilon}} \rightarrow \hat{\boldsymbol{\sigma}} \rightarrow \mathbf{X}, \quad \mathbf{u} \in\{\{w, 0,0\},\{0, w, 0\},\{0,0, w\}\}
$$

где $\mathbf{u}, \mathbf{X}$ - векторы перемещений и сил; $\hat{\boldsymbol{\varepsilon}}, \hat{\boldsymbol{\sigma}}$ - тензоры деформаций и напряжений. Если через $k$ обозначить порядок полиномиального вектора $\mathbf{X}$, то $\alpha+\beta+\gamma-2=k$. Каждому полиномиальному внутреннему состоянию $\xi_{p}=\{\mathbf{u}, \hat{\varepsilon}, \hat{\boldsymbol{\sigma}}\}$ соответствует вектор $\mathbf{X}_{p}$, компонентами которого являются однородные полиномы порядка $k$.

Например, изотропной эластостатической цепочке (1) соответствуют определяющие соотношения [3]:

$$
\hat{\boldsymbol{\varepsilon}}=\frac{1}{2}\left[(\nabla \mathbf{u})^{\top}+\nabla \mathbf{u}\right], \quad \hat{\boldsymbol{\sigma}}=\lambda \boldsymbol{\Theta} \hat{\mathbf{E}}+2 \mu \hat{\boldsymbol{\varepsilon}}, \quad \mathbf{X}=-\operatorname{div} \hat{\boldsymbol{\sigma}}
$$

где $\hat{\mathbf{E}}$ - единичный тензор; $\boldsymbol{\Theta}=I_{1}(\hat{\boldsymbol{\varepsilon}})$ - первый инвариант тензора деформаций (объемная деформация); $\lambda, \mu$ - параметры Ламе $\left(\lambda=\frac{2 \nu \mu}{1-2 \nu}, \nu\right.$ - коэффициент Пуассона).

Ранее используемые подходы к разложению вектора $\mathbf{X}$ по элементам отрезка базиса $\left\{\mathbf{X}_{k}\right\}$ максимального порядка $K, k \in\{0,1,2, \ldots, K\}$, опирались на применение метода наименьших квадратов или рядов Фурье. В обоих случаях использовалось скалярное произведение

$$
\left(\mathbf{X}_{k}, \mathbf{X}_{j}\right)=\int_{V_{0}} \mathbf{X}_{k} \cdot \mathbf{X}_{j} d V
$$

где $V_{0}$ не обязано совпадать с $V$ и может иметь более тривиальную форму для цели экономии ресурсов, например, куб:

$$
V_{0}=\left\{(x, y, z) \in \mathbb{R}^{3},-1 \leqslant x, y, z \leqslant 1\right\} .
$$

Это обстоятельство обусловлено регулярностью элементов базиса не только внутри $V$, но также и в любой ограниченной области благодаря именно полиномиальному характеру представления.

Практика проведения расчетов показала, что в некоторых задачах (нелинейные среды, декомпозируемые применением метода возмущений в последовательность линейных, но содержащих искусственно порожденную полиномиальную объемную нагрузку) для удовлетворительного приближения частного решения относительно состояний от объемных сил требуется достаточно большой порядок $K$ аппроксимирующих многочленов, из-за чего вычисление $K^{2}$ интегралов даже в «канонических» областях требует приличных затрат времени. Проблема была бы снята, если бы опорный базис имел не полиномиальный, а более узкий, мономный характер. Это предполагает, что вектор $\mathbf{X}^{k}$ содержит моном порядка $k$ ровно в одной позиции:

$$
\mathbf{X}^{k} \in\left\{\left\{w_{X}, 0,0\right\},\left\{0, w_{X}, 0\right\},\left\{0,0, w_{X}\right\}\right\}, \quad w_{X}=x^{a} y^{b} z^{c}, \quad a+b+c=k .
$$


Действительно, любой однородный полиномиальный вектор $\mathbf{X}$ является заданной линейной комбинацией элементов базиса $\left\{\mathbf{X}^{k}\right\}$ :

$$
\mathbf{X}=\sum_{k=0}^{K} \chi_{k} \mathbf{X}^{k}
$$

Если при этом каждому элементу $\mathbf{X}^{k}$ соответствует состояние $\xi^{k}$ (которое надо построить), то можно предположить аналогичное разложение для внутреннего состояния $\xi^{*}$ от объемных сил:

$$
\xi^{*}=\sum_{k=0}^{K} \chi_{k} \xi^{k}
$$

Предположение не оказывается ограничивающим, его доказывать нет необходимости, поскольку расчетчика устраивает любое частное решение $\xi^{*}$, лишь бы выполнялось линейное отображение $\xi^{*} \rightarrow \mathbf{X}$. Последнее гарантировано цепочкой (1).

Таким образом, возникла задача о формировании мономного базиса $\left\{\mathbf{X}^{k}\right\}$ на основе опорного однородно-полиномиального базиса $\left\{\mathbf{X}_{k}\right\}$.

\section{2. Алгоритм формирования внутренних состояний для мономно-} го базиса объемных сил. Совокупность мономов порядка $k$ будем называть «кластером $k »$. Число различных мономов $w_{k}=x^{a} y^{b} z^{c}, a+b+c=k$ определяет мощность этого кластера

$$
n_{k}=\frac{1}{2}(k+1)(k+2) \text {. }
$$

Поскольку каждый из мономов может быть помещен в любую позицию трехмерного вектора $\mathbf{X}^{k}$, количество различных векторов этого порядка оценивается как $N_{k}=3 n_{k}=\frac{3}{2}(k+1)(k+2)$. Каким-либо способом введем индивидуальную нумерацию всех элементов мономного отрезка базиса векторов $\mathbf{X}^{k}$, например, в соответствии с табл. 1. После этого множество номеров элементов кластера принадлежит списку $i \in\left\{1,2, \ldots, N_{k}\right\}$.

При формировании отрезка опорного базиса $\left\{\mathbf{X}_{k}\right\}$ в соответствии с принятым обозначением (табл. 1) каждый элемент опорного базиса приобретает свой индекс $i$ и представляется линейной комбинацией

$$
\mathbf{X}_{i}=\sum_{j=1}^{N_{K}} c_{i j} \mathbf{X}^{j}
$$

Таблица 1

Внутрикластерная нумерация элементов мономного базиса

[Intracluster numbering of elements of a monomic basis]

\begin{tabular}{c|r|r|r|l|r}
\hline position of $w_{k}$ & \multicolumn{1}{|c|}{$x^{k}$} & $x^{k-1} y$ & $x^{k-2} y^{2}$ & $\ldots$ & $z^{k}$ \\
\hline 1 & 1 & 2 & 3 & $\ldots$ & $n_{k}$ \\
2 & $n+1$ & $n+2$ & $n+3$ & $\ldots$ & $2 n_{k}$ \\
3 & $2 n+1$ & $2 n+2$ & $2 n+3$ & $\ldots$ & $3 n_{k}$ \\
\hline
\end{tabular}


или в матричной форме (индексом $k$ матрицу $C$ помечать нет необходимости: она используется внутрикластерно):

$$
\left\{\mathbf{X}_{i}\right\}=C\left\{\mathbf{X}^{j}\right\}, \quad C=\left[c_{i j}\right], \quad i, j \in\left\{1,2, \ldots, N_{k}\right\}
$$

Все коэффициенты матрицы $C$ определены, причем $\operatorname{det} C \neq 0$ в силу линейной независимости элементов опорного базиса. Обращение матрицы $C$ позволяет получить выражение элементов мономного базиса $\left\{\mathbf{X}^{j}\right\}$ через уже известное представление опорного базиса в виде

$$
\left\{\mathbf{X}^{j}\right\}=C^{-1}\left\{\mathbf{X}_{i}\right\}
$$

что нам и требовалось.

Әти же схемы позволяют назначить базис полиномиальных внутренних состояний:

$$
\xi^{j}=\sum_{i=1}^{N_{K}} d_{j i} \xi_{i},
$$

где $d_{j i}$ - элементы матрицы $D=C^{-1}=\left[d_{j i}\right]$.

Перебирая поочередно кластеры до их максимального значения, накапливаем последовательность внутренних состояний $\left\{\xi^{l}\right\}$. Поскольку полиномиальный вектор объемных сил $\mathbf{X}^{*}$ известен и является линейной комбинацией элементов базиса

$$
\mathbf{X}^{*}=\sum_{l} \chi_{l} \mathbf{X}^{l}
$$

ему соответствующее внутреннее состояние определяется аналогично:

$$
\xi^{*}=\sum_{l} \chi_{l} \xi^{l}
$$

В выражениях (4), (5) нумерация элементов базиса изменена в соответствии с фактом накопления базиса списками $\left\{\mathbf{X}^{j}\right\}$; таких списков столько же, сколько имеется различных кластеров.

Ниже приводится более детальное описание алгоритма формирования внутреннего состояния, строго соответствующее полиномиальной объемной силе $\mathbf{X}$.

А. ВНУтРИКЛАСТЕРНЫЕ оПЕРАции. Входной информацией для блока служит порядок кластера $k$ и однородный полиномиальный вектор $\mathbf{X}$.

А.1. Определяются мощность $n_{k}$ множества мономов порядка $k$ и размерность $N_{k}$ матрицы $C$.

А.2. Перебираются позиции $p \in\{1,2,3\}$ для формирования мономного базиса $\left\{\mathbf{X}^{j}\right\}$. Полагаем $j=0$. Осуществляется перебор порядков степеней переменных $x, y, z$ в соответствии с табл. 1:

$$
\forall c \in\left\{0,1, \ldots, n_{k}\right\}: \forall b \in\left\{0,1, \ldots, n_{k}-c\right\}: a=n_{k}-c-b ; j:=j+1 .
$$

Формируется моном $w_{x}=x^{a} y^{b} z^{c}$ и вектор $\mathbf{X}^{j}$ в соответствии с позицией $p$. 
Использование «сортировочного алгоритма» [20] позволяет для каждого набора параметров $a, b, c, p$ указать значения $\alpha, \beta, \gamma$ и сформировать вектор $\mathbf{X}_{i}$, являющийся линейной комбинацией (3) элементов мономного базиса. Определяются все коэффициенты $c_{i j}$ строки $i$ матрицы $C$. По завершении перебора (6) матрица $C$ сформирована.

А.3. Выполняется обращение матрицы $C: C^{-1}=D=\left[d_{j i}\right]$; тем самым список элементов опорного базиса $\left\{\mathbf{X}_{i}\right\}$ представляется линейной комбинацией элементов мономного базиса $\left\{\mathbf{X}^{j}\right\}$ :

$$
\left\{\mathbf{X}_{i}\right\}=D\left\{\mathbf{X}^{j}\right\}, \quad \mathbf{X}_{i}=\sum_{j} x_{j i} \mathbf{X}^{j}
$$

А.4. Входной кластерный вектор объемных сил $\mathbf{X}$ представляется линейной комбинацией элементов мономного базиса

$$
\mathbf{X}=H\left\{\mathbf{X}^{j}\right\}, \quad \mathbf{X}=\sum_{j} \chi_{j} \mathbf{X}^{j} .
$$

Здесь $H=\left\{\chi_{j}\right\}$ - вектор-строка коэффициентов разложения (2) размерности $N_{k}$.

А.5. Осуществляется перебор элементов мономного базиса. Изначально полагается значение внутреннего состояния среды нулевым: $\xi^{*}=0$.

Номеру $j$ элемента мономного базиса соответствуют номер $i=j$ элемента опорного базиса и отвечающие ему значения $\alpha, \beta, \gamma$. «ортировочный алгоритм» формирует состояние $\xi_{i}^{*}=\left\{\mathbf{u}_{i}, \hat{\boldsymbol{\varepsilon}}_{i}, \hat{\boldsymbol{\sigma}}_{i}\right\}$, причем такую процедуру необходимо делать в том случае, если коэффициент $b_{i}=\sum_{j} \chi_{j} x_{j i}$ матрицы $B=H K=\left\{b_{i}\right\}$ не равен нулю (экономия ресурсов).

В процессе перебора осуществляется накопление внутреннего состояния $\xi^{*}$ для кластера $k$ :

$$
\xi^{*}:=\xi^{*}+\xi_{i}^{*}
$$

По окончании цикла внутреннее состояние $\xi^{*}$, отвечающее однородному полиномиальному вектору $\mathbf{X}$, построено.

В. ВНЕКЛАСТЕРНЫЕ ОПЕРАЦИИ.

В.0. Определяется максимальный порядок $K_{\max }$ полиномов вектора объемных сил $\mathbf{F}_{0}$. Приводится его представление $\mathbf{F}_{0}$ в форме линейной комбинации однородных полиномиальных векторов $\mathbf{f}_{k}$ :

$$
\mathbf{F}_{0}=\sum_{k=0}^{K_{\max }} \mathbf{f}_{k}
$$

где $k$ далее обозначает порядок кластера.

Устанавливается нулевое значение для накапливаемых внутреннего состояния $\xi=0$ вектора сил $(\mathbf{F}=0)$.

В.1. Осуществляется перебор кластеров в порядке возрастания: $\forall k \in\{0,1, \ldots$, $\left.K_{\max }\right\}$; выделяется вектор $\mathbf{X}=\mathbf{f}_{k}$ и выполняются внутрикластерные операции пункта $A$.

Результаты внутрикластерных операций накапливаются:

$$
\xi:=\xi+\xi^{*}, \quad \mathbf{F}:=\mathbf{F}+\mathbf{X} .
$$


В.2. Выполняется проверка точности построения внутреннего состояния, отвечающего приложенным объемным силам. Это легче всего сделать на основе оценки нормы вектора:

$$
\left\|\mathbf{F}_{0}-\mathbf{F}\right\| \rightarrow \min
$$

Процесс построения напряженно-деформированного состояния, отвечающего внешним объемным силам, завершен.

ЗАмечАниЕ. Все операции в алгоритме дают однозначное представление результата. Если описывать исходную числовую информацию в рациональной форме, то окончательный результат также имеет однозначно определяемую рациональную форму. Следовательно, для заданного вектора объемных сил указывается строго соответствующее ему внутреннее состояние $\xi^{*}$.

3. Примеры построения напряженно-деформированных состояний тел от объемных сил. Ниже приведены примеры восстановления напряженно-деформированного состояния односвязных эластостатических тел, порожденного объемными силами полиномиального характера.

\section{1. Тестирование алгоритма восстановления напряженно-дефор- мированного состояния, строго соответствующего объемной силе.} Процедура восстановления строгого решения задачи о напряженно-деформированном состоянии односвязного линейно-упругого тела, вызванном полиномиальными силами, была успешно проверена на объектах довольно высокого 13-го порядка полиномов и убедила в факте строгого построения соответствующего внутреннего состояния.

В качестве примера приводим вариант задачи, результаты решения которой имеют компактный характер. Пусть односвязное тело находится под воздействием объемной силы

$$
\mathbf{X}=\left(\begin{array}{c}
1+x y \\
-6 x^{2} z \\
2 x y z
\end{array}\right)
$$

разложенной в сумму слагаемых четырех кластеров $\mathbf{X}^{\langle k\rangle}$ :

$$
\begin{gathered}
\mathbf{X}=\sum_{k=0}^{3} \mathbf{X}^{\langle k\rangle}, \\
\mathbf{X}^{\langle 0\rangle}=\left(\begin{array}{l}
1 \\
0 \\
0
\end{array}\right), \quad \mathbf{X}^{\langle 1\rangle}=\left(\begin{array}{l}
0 \\
0 \\
0
\end{array}\right), \quad \mathbf{X}^{\langle 2\rangle}=\left(\begin{array}{c}
x y \\
0 \\
0
\end{array}\right), \quad \mathbf{X}^{\langle 3\rangle}=\left(\begin{array}{c}
0 \\
-6 x^{2} z \\
2 x y z
\end{array}\right) .
\end{gathered}
$$

C каждым слагаемым этой суммы выполнялись внутрикластерные операции, рационально строились соответствующие внутренние состояния. Накопленное суммарно внутреннее состояние $\xi=\sum_{k} \xi^{*\langle k\rangle}$ дало результат

$$
\mathbf{u}=\left(\begin{array}{c}
-\frac{1}{18} x^{3} y-\frac{1}{2} z^{2}+\frac{1}{18} y z^{4} \\
\frac{1}{36} x^{4}+\frac{1}{2} x^{4} z+\frac{1}{18} x z^{4} \\
-\frac{1}{9} x y z^{3}
\end{array}\right)
$$




$$
\begin{gathered}
\hat{\varepsilon}=\left(\begin{array}{ccc}
-\frac{1}{6} x^{2} y & \frac{1}{36} x^{3}+x^{3} z+\frac{1}{18} z^{4} & -\frac{1}{2} z+\frac{1}{18} y z^{3} \\
\frac{1}{36} x^{3}+x^{3} z+\frac{1}{18} z^{4} & 0 & \frac{1}{4} x^{4}+\frac{1}{18} x z^{3} \\
-\frac{1}{2} z+\frac{1}{18} y z^{3} & \frac{1}{4} x^{4}+\frac{1}{18} x z^{3} & -\frac{1}{3} x y z^{2}
\end{array}\right), \\
\hat{\sigma}=\left(\begin{array}{ccc}
-\frac{1}{2} x^{2} y-\frac{1}{3} x y z^{2} & \frac{1}{18} x^{3}+2 x^{3} z+\frac{1}{9} z^{4} & -z+\frac{1}{9} y z^{3} \\
\frac{1}{18} x^{3}+2 x^{3} z+\frac{1}{9} z^{4} & -\frac{1}{6} x^{2} y-\frac{1}{3} x y z^{2} & \frac{1}{2} x^{4}+\frac{1}{9} x z^{3} \\
-z+\frac{1}{9} y z^{3} & \frac{1}{2} x^{4}+\frac{1}{9} x z^{3} & -\frac{1}{6} x^{2} y-x y z^{2}
\end{array}\right) .
\end{gathered}
$$

При этом цепочка действий (1) привела в точности к состоянию (7), что подчеркнуло строгий характер полученного решения.

3.2. Состояние шара, находящегося под действием центральных сил и сил инерции. В качестве расчетного примера рассматривается однородный шар безразмерного радиуса 1, находящегося под действием центральных сил (силы типа удельного веса в плоти Земли) и сил инерции, вызванных вращением шара вокруг оси $S N$ (см. рисунок, $\omega-$ угловая скорость).

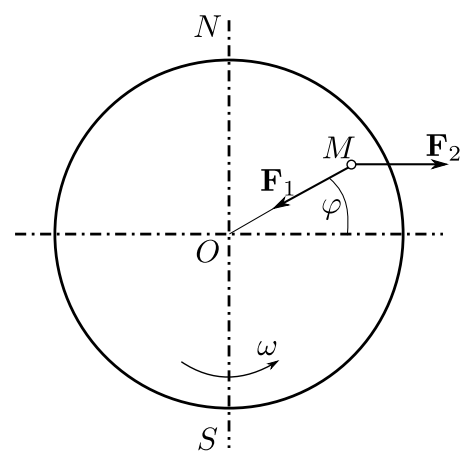

Схема нагружения однородного шара объемными силами

[Scheme of loading a homogeneous ball with bulk forces]

Будем полагать положение точки $M$ описываемым радиус-вектором $\mathbf{r}$ в центральной системе координат $O x y z$. Величины сил $\mathbf{F}_{1}, \mathbf{F}_{2}$ зависят от физико-геометрических параметров объекта. После обезразмеривания считаем их характеризуемыми константами $f_{1}, f_{2}$ соответственно:

$$
\mathbf{F}_{1}=-f_{1}\left(\begin{array}{c}
x \\
y \\
z
\end{array}\right), \quad \mathbf{F}_{2}=f_{2}\left(\begin{array}{l}
x \\
y \\
0
\end{array}\right)
$$

так что их сумма

$$
\mathbf{X}=\mathbf{F}_{1}+\mathbf{F}_{2}=\left(\begin{array}{c}
\left(f_{2}-f_{1}\right) x \\
\left(f_{2}-f_{1}\right) y \\
-f_{1} z
\end{array}\right)
$$

и определяет внутреннюю объемную силу шара.

Интерес представляет модификация внутреннего состояния при различных соотношениях параметров $f_{1}, f_{2}$. В табл. 2 приведены результаты обследования трех ярко выраженных состояний: 
1) медленное вращение: $f_{2} \ll f_{1}$ (положено $f_{2}=0$ );

2) компенсационное вращение: $f_{2} \approx f_{1}$ (положено $f_{2}=f_{1}$ );

3) быстрое вращение $f_{2} \gg f_{1}$ (положено $f_{1}=0$ ).

В табл. 2 приведены качественные картины распределения напряжений в радиальном сечении однородного упругого тела с коэффициентом Пуассона $\nu=1 / 4$ (первый квадрант сечения в плоскости Оxyz). Фон за пределами четверти круга соответствует нулевому уровню соответствующих напряжений, более светлые тона отвечают большим напряжениям (при растяжении соответствующих волокон - нормальным напряжениям; при сужении

Таблица 2

Напряженное состояние гравитационного вращающегося тела

[Stress state of a gravitational rotating body]

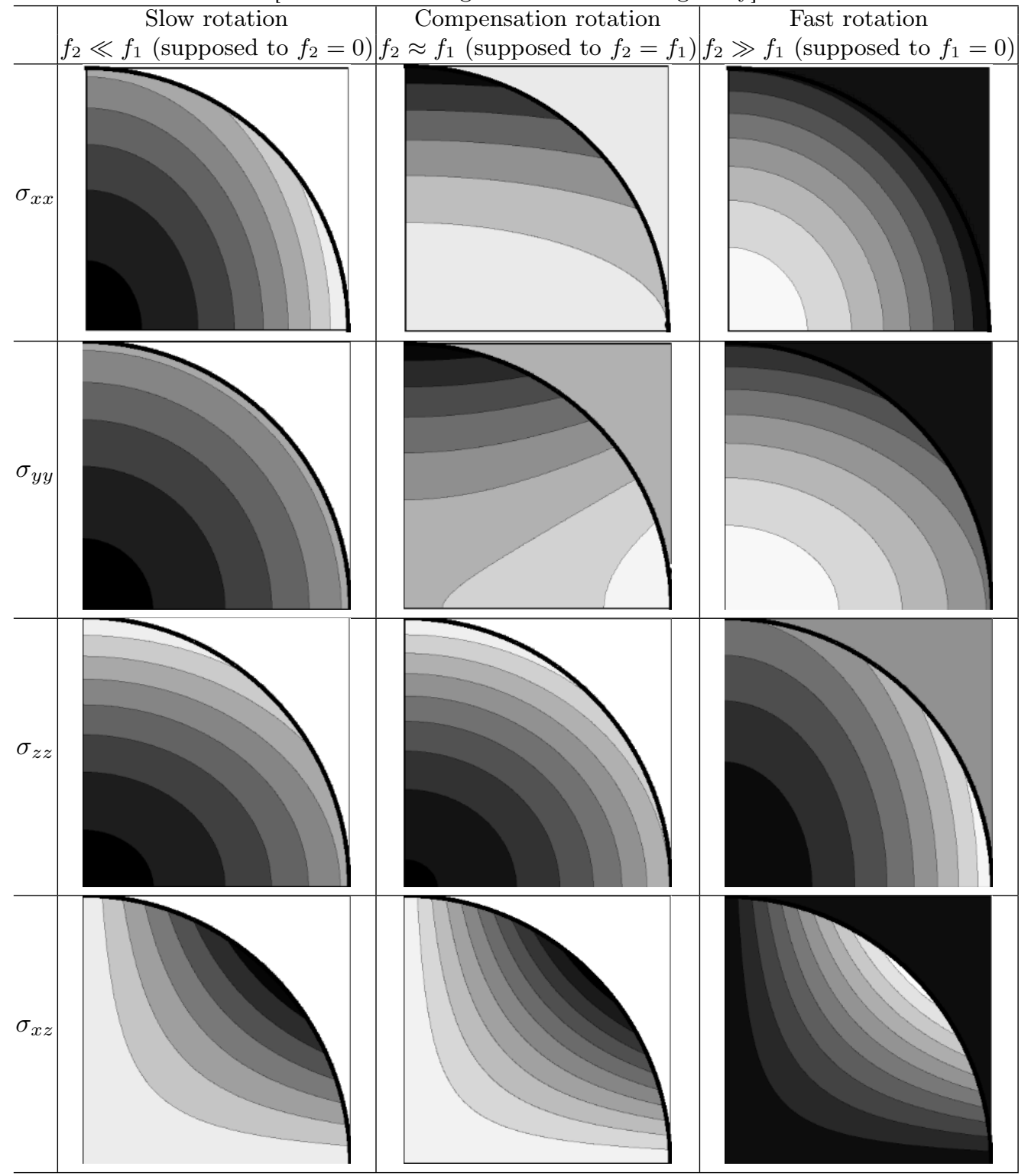


угла $x O z$ - напряжениям сдвига $\left.\sigma_{x z}\right)$. При комментировании рисунков будем употреблять термины, принятые при описании геометрии Земного шара экваториальное сечение, полярная ось $S N$, широта. Вследствие непрерывной зависимости решения от значения коэффициента $\nu$ будем допускать прогнозы при его малой вариации.

При медленном вращении шара наибольшее сжатие материала тела происходит в его центре. Расширение углов сдвиговых деформаций наибольшее в средних широтах на поверхности шара. В реальности для слабо сжимаемых тел здесь следует ожидать эффектов, ориентирующихся на хрупкое разрушение, образование трещин (то же при компенсационном вращении).

При компенсационном вращении, когда силы инерции частично уравновешивают гравитационные силы, радиальные волокна вблизи экваториальной плоскости практически не деформируются, а при приближении к полюсам $S N$ ожидается их сжатие. Вблизи полюсов также сжимаются окружные волокна, а вот вблизи поверхности шара в «граничных» зонах они, наоборот, растягиваются.

При быстром вращении радиальные и окружные волокна значительно растягиваются вблизи центра шара, в то время как осевые волокна в этой области, наоборот, укорачиваются. На большей части поверхности (чем ближе к экватору, тем сильнее) осевые волокна, напротив, удлиняются. При очень быстром вращении «мягких» материалов это может служить причиной их стремления к образованию тороидальных тел (на геометрически линейном уровне это констатировать не удается). В средних широтах поверхности шара происходит сужение межволоконных углов, что препятствует образованию радиальных трещин хрупких сред в этих местах. В этом состоит принципиальное отличие от медленных и компенсационных вращений.

\section{Выводы.}

1. Теоретически обеспечены процедуры восстановления внутреннего состояния односвязного линейно-упругого тела при квазистатическом нагружении полиномиальными объемными силами произвольного физического смысла.

2. Построен и реализован в системе Mathematica эффективный алгоритм весьма быстрого восстановления напряженно-деформированного состояния, отвечающего объемным силам. Время счета измеряется минутами; при использовании энергетических методов затрачиваются часы.

3. Выполнено тестирование указанных процедур для полиномиальных объемных сил высокой степени многочленов, что гарантирует его эффективное применение даже в задачах нелинейной теории упругости, в которых нелинейные соотношения раскладываются средствами метода возмущений в последовательность линейных с традиционно искусственно порождаемыми объемными силами именно полиномиального характеpa.

4. Эффективность алгоритма продемонстрирована на задаче о вращении однородного гравитационного шара при различных соотношениях параметров гравитации и вращения. Это позволило сделать ряд качественных выводов о состоянии тела.

Ближайшей перспективой развития подхода к восстановлению напряженно-деформированного состояния от объемных сил является расширение об- 
ласти его применения на однополостные и многополостные ограниченные и неограниченные тела. Технически такой приближенный подход уже реализуется [21], но соответствующее обоснование еще не выполнено. Еще более весомым явился бы результат, дающий строгое решение таких задач.

Конкурирующие интересы. Конкурирующих интересов не имеем.

Авторский вклад и ответственность. Все авторы принимали участие в разработке концепции статьи; все авторы сделали эквивалентный вклад в подготовку публикации. Авторы несут полную ответственность за предоставление окончательной рукописи в печать. Окончательная версия рукописи была одобрена всеми авторами.

Финансирование. Исследование выполнено при финансовой поддержке РФФИ и Липецкой области в рамках научного проекта № 19-41-480003 p_a.

\section{Библиографический список}

1. Truesdell C. A First Course in Rational Continuum Mechanics. Vol. 1: General Concepts / Pure and Applied Mathematics. vol. 71. New York, San Francisco, London: Academic Press, 1977. xxiii+280 pp.

2. Работнов Ю. Н. Механика деформируемого твердого тела. М.: Наука, 1988.712 с.

3. Лурье А. И. Теория упругости. М.: Наука, 1970. 940 с.

4. Мусхелишвили Н. И. Некоторые основные задачи математической теории упругости. М.: Наука, 1966. 707 с.

5. Green A. E., Zerna W. Theoretical Elasticity. New York: Dover Publications, 1992. $\mathrm{xvi}+457 \mathrm{pp}$.

6. Arfken G. B., Weber H. J. Mathematical Methods for Physicists. Amsterdam: Elsiver/Academic Press, 2005. xii+1182 pp.

7. Neuber H. Ein neuer Ansatz zur Lösung räumlicher Probleme der Elastizitätstheorie. Der Hohlkegel unter Einzellast als Beispiel // ZAMM, 1934. vol. 14, no. 4. pp. 203-212. https:// doi.org/10.1002/zamm. 19340140404.

8. Матвеенко В. П., Шевелев Н. А. Аналитическое исследование напряженнодеформированного состояния тел вращения, находящихся под действием массовых сил / Напряженно-деформированное состояние конструкций из упругих и вязкоупругих материалов. Свердловск: УНЦ АН СССР, 1977. С. 54-60.

9. Вестяк В. А., Тарлаковский Д. В. Нестационарное осесимметричное деформирование упругого пространства со сферической полостью под действием объемных сил // Вестник Московского университета. Сер. 1. Математика. Механика, 2016. Т. 71 , № 4 . C. $48-54$.

10. Шарафутдинов Г. З. Функции комплексного переменного в задачах теории упругости при наличии массовых сил // ПММ, 2009. Т. 73, № 1. С. 69-87.

11. Зайцев А. В., Фукалов А. А. Точные аналитические решения задач о равновесии упругих анизотропных тел с центральной и осевой симметрией, находящихся в поле гравитационных сил, и их приложения к задачам геомеханики // Maтематическое моделирование в естественных науках, 2015. Т. 1. С. 141-144.

12. Пикуль В. В. К аномальному деформированию твердых тел// Физическая мезомеханика, 2013. Т. 16, № 2. С. 93-100.

13. Козлов В. В. Сила Лоренца и ее обобщения // Нелинейная динам., 2011. Т. 7 , №3. C. $627-634$.

14. Саталкина Л. В. Метод граничных состояний в задачах теории упругости неоднородных тел и термоупругости: Дис. . . канд. физ.-мат. наук. Липецк, 2010. 108 с.

15. Penkov V. B., Novikov E. A., Novikova O. S., Levina L. V. Combining the method of boundary states and the Lindstedt-Poincaré method in geometrically nonlinear elastostatics // J. Phys.: Conf. Ser., 2020. vol. 1479, 012135. https://doi.org/10.1088/1742-6596/ 1479/1/012135. 
16. Иванычев Д. А., Новиков Е. А. Решение физически нелинейных задач для изотропных тел методом граничных состояний / Проблемы прочности, пластичности и устойчивости в механике деформируемого твердого тела. Тверь: ТвГТУ, 2021. С. 43-47.

17. Penkov V. B., Ivanychev D. A., Levina L. V., Novikov E. A. Using the method of boundary states with perturbations to solve physically nonlinear problems of the theory of elasticity // J. Phys.: Conf. Ser., 2020. vol. 1479, 012134. https://doi.org/10.1088/1742-6596/1479/ $1 / 012134$.

18. Кузьменко В. И., Кузьменко Н. В., Левина Л. В., Пеньков В. Б. Способ решения задач изотропной теории упругости с объемными силами в полиномиальном представлении // ПММ, 2019. Т. 83, № 1. С. 84-94. https ://doi .org/10.1134/S0032823519010053.

19. Пеньков В. Б., Левина Л. В., Новикова О. С. Аналитическое решение задач эластостатики односвязного тела, нагруженного неконсервативными объемными силами. Теоретическое и алгоритмическое обеспечение // Вестн. Сам. гос. техн. ун-та. Сер. Физ.мат. науки, 2020. Т. 24, №1. С. 56-73. https://doi.org/10.14498/vsgtu1711.

20. Иванычев Д. А. Метод граничных состояний при решении смешанной задачи теории анизотропной упругости с массовыми силами // Вестн. Томск. гос. ун-та. Матем. и мех., 2021. № 71. С. 63-77. https ://doi .org/10.17223/19988621/71/6.

21. Пеньков В. Б., Рыбакова М. Р., Саталкина Л. В. Применение алгоритма Шварца к пространственным задачам теории упругости // Вести вузов Черноземъя, 2015. № 2 (40). C. $23-31$. 


\title{
MSC: 74A10
}

\section{Rigorous solution of the problem of the state of a linearly elastic isotropic body under the action of polynomial bulk forces}

\author{
(C) V. B. Penkov, L. V. Levina, E. A. Novikov
}

Lipetsk State Technical University,

30, Moskovskaya st., 398055, Russian Federation.

\begin{abstract}
When solving boundary value problems about the construction of the stress-strain state of an linearly elastic, isotropic body, an important step is finding the internal state generated by the forces, distributed over the area occupied by the body. In the classical version, there is a numerical method for estimating the state at any point of the body based on the singular-integral representation of Cesaro. In the variant of conservative bulk forces, it is possible to construct solutions in an analytical form. With arbitrary regular effects of mechanical and other physical nature the force is not potential and the approaches of Papkovich-Neiber and Arzhanykh-Slobodyansky are powerless. In addition, the solution of nonlinear problems of elastostatics by means of the perturbation method, as well as the use of the Schwarz algorithm in solving problems for the study of multi-cavity solids, lead to the need to solve a sequence of linear problems. At the same time, fictitious bulk forces are necessarily generated, which as a rule have a polynomial nature.

The method proposed by the authors earlier for estimating the stressstrain state of a solid caused by the action of polynomial bulk forces represented in Cartesian coordinates has been improved. The internal state is restored in strict accordance with the forces statically acting on a simply connected bounded linear-elastic body. An effective method for constructing a solution and an algorithm for its computer implementation are proposed and described. Test calculations are demonstrated. The analysis of the state of the ball under the action of a superposition of bulk forces of different nature at different ratios of parameters that emphasize the level of influence of
\end{abstract}

\section{Research Article}

( () (1) The content is published under the terms of the Creative Commons Attribution 4.0 International License (http://creativecommons.org/licenses/by/4.0/)

Please cite this article in press as:

Penkov V. B., Levina L. V., Novikov E. A. Rigorous solution of the problem of the state of a linearly elastic isotropic body under the action of polynomial bulk forces, Vestn. Samar. Gos. Tekhn. Univ., Ser. Fiz.-Mat. Nauki [J. Samara State Tech. Univ., Ser. Phys. Math. Sci.], 2021, vol. 25, no. 3, pp. 475-490. https://doi.org/10.14498/vsgtu1864 (In Russian).

\section{Authors' Details:}

Viktor B. Penkov (10) https://orcid.org/0000-0002-6059-1856

Dr. Phys. \& Math. Sci.; Professor; Dept. of General Mechanics; e-mail: vbpenkov@mail .ru

Lyubov V. Levina (D) https://orcid.org/0000-0002-7441-835X

Cand. Phys. \& Math. Sci.; Associate Professor; Dept. of Applied Mathematics;

e-mail: satalkina_lyubov@mail.ru

Evgeny A. Novikov (1) https://orcid.org/0000-0001-5606-5207

Postgraduate Student; Dept. of General Mechanics; e-mail: 89513027802@mail.ru 
these factors is performed. The results are presented graphically. Conclusions are drawn:

a) the procedure for writing out the stress-strain state on the volume forces represented by polynomials from Cartesian coordinates is justified;

b) the algorithm is implemented in the Mathematica computing system and tested on high-order polynomials;

c) the analysis of the quasi-static state of a linear-elastic isotropic ball exposed to the forces of gravity and inertia at various combinations of parameters corresponding to the variants of slow, fast, compensatory (inertial forces are proportional to the gravitational) rotations is carried out.

The prospects for the development of a new approach to the class of bounded and unbounded bodies containing an arbitrary number of cavities are noted.

Keywords: bulk forces, partial solution, stress-strain state from bulk forces, linear elastostatics, elasticity theory, partial solution of the Lame equations.

Received: $22^{\text {nd }}$ April, $2021 /$ Revised: $7^{\text {th }}$ September, $2021 /$

Accepted: $20^{\text {th }}$ September, $2021 /$ First online: $30^{\text {th }}$ September, 2021

Competing interests. The authors declare no conflicts of interests.

Authors' contributions and responsibilities. Each author has participated in the article concept development; the authors contributed equally to this article. The authors are absolutely responsible for submit the final manuscript to print. Each author has approved the final version of manuscript.

Funding. The research was supported by the Russian Foundation for Basic Research and the Lipetsk Region (grant no. 19-41-480003 r_a).

\section{References}

1. Truesdell C. A First Course in Rational Continuum Mechanics. Vol. 1: General Concepts, Pure and Applied Mathematics, vol. 71. New York, San Francisco, London, Academic Press, 1977, xxiii +280 pp.

2. Rabotnov Yu. N. Mekhanika deformiruemogo tverdogo tela [Mechanics of a Deformable Rigid Body]. Moscow, Nauka, 1988, 712 pp. (In Russian)

3. Lurie A. I. Theory of Elasticity, Foundations of Engineering Mechanics. Berlin, SpringerVerlag, 2005, iv+1050 pp. https://doi .org/10.1007/978-3-540-26455-2.

4. Muskhelishvili N. I. Nekotorye osnovnye zadachi matematicheskoi teorii uprugosti [Some Basic Problems of the Mathematical Theory of Elasticity]. Moscow, Nauka, 1966, 707 pp. (In Russian)

5. Green A. E., Zerna W. Theoretical Elasticity. New York, Dover Publications, 1992, $\mathrm{xvi}+457 \mathrm{pp}$.

6. Arfken G. B., Weber H. J. Mathematical Methods for Physicists. Amsterdam, Elsiver/Academic Press, 2005, xii+1182 pp.

7. Neuber H. Ein neuer Ansatz zur Lösung räumlicher Probleme der Elastizitätstheorie. Der Hohlkegel unter Einzellast als Beispiel, ZAMM, 1934, vol. 14, no. 4, pp. 203-212. https:// doi.org/10.1002/zamm.19340140404.

8. Matveenko V. P., Schevelev N. A. Analytical study of the stress-strain state of rotation bodies under the action of mass forces, In: Stress-Strain State of Structures Made of Elastic and Viscoelastic Materials. Sverdlovsk, 1977, pp. 54-60 (In Russian).

9. Vestyak V. A., Tarlakovsky D. V. Unsteady axisymmetric deformation of an elastic space with a spherical cavity under the action of bulk forces, Moscow Univ. Mech. Bull., 2016, vol. 71, no. 4, pp. 87-92. https://doi.org/10.3103/S0027133016040038. 
10. Sharafutdinov G. Z. Functions of a complex variable in problems in the theory of elasticity with mass forces, J. Appl. Math. Mech., 2009, vol.73, no.1, pp. 69-87. https://doi.org/ 10.1016/j.jappmathmech.2009.03.008.

11. Zaytsev A. V, Fukalov A. A. Exact analytical solutions of equilibrium problems for elastic anisotropic bodies with central and axial symmetry, which are in the field of gravitational forces, and their applications to the problems of geomechanics, Matemat. Model. Estestv. Nauk., 2015, vol. 1, pp. 141-144 (In Russian).

12. Pikul V. V. To anomalous deformation of solids, Physical Mesomechanics, 2013, no. 2, pp. 93-100 (In Russian).

13. Kozlov V. V. The Lorentz force and its generalizations, Nelin. Dinam., 2011, vol. 7, no. 3, pp. 627-634 (In Russian).

14. Satalkina L. V. The method of boundary states in problems of the theory of elasticity of inhomogeneous bodies and thermoelasticity, Thesis of Dissertation (Cand. Phys. \& Math. Sci.). Lipetsk, 2010, 108 pp. (In Russian)

15. Penkov V. B., Novikov E. A., Novikova O. S., Levina L. V. Combining the method of boundary states and the Lindstedt-Poincaré method in geometrically nonlinear elastostatics, J. Phys.: Conf. Ser., 2020, vol.1479, 012135. https://doi.org/10.1088/1742-6596/ 1479/1/012135.

16. Ivanychev D. A., Novikov E. A. The solution of physically nonlinear problems for isotropic bodies by the method of boundary states, In: Problems of Strength, Plasticity, and Stability in Solid Mechanics. Tver, Tver State Techn. Univ., 2021, pp. $43-47$ (In Russian).

17. Penkov V. B., Ivanychev D. A., Levina L. V., Novikov E. A. Using the method of boundary states with perturbations to solve physically nonlinear problems of the theory of elasticity, J. Phys.: Conf. Ser., 2020, vol. 1479, 012134. https://doi.org/10.1088/1742-6596/1479/ $1 / 012134$.

18. Kuzmenko V. I., Kuzmenko N. V., Levina L. V., Penkov V. B. A method for solving problems of the isotropic elasticity theory with bulk forces in polynomial representation, Mech. Solids, 2019, vol.54, no. 5, pp. 741-749. https://doi.org/10.3103/S0025654419050108.

19. Penkov V. B., Levina L. V., Novikova O. S. Analytical solution of elastostatic problems of a simply connected body loaded with nonconservative volume forces: theoretical and algorithmic support, Vestn. Samar. Gos. Tekhn. Univ., Ser. Fiz.-Mat. Nauki [J. Samara State Tech. Univ., Ser. Phys. Math. Sci.], 2020, vol. 24, no. 1, pp. 56-73 (In Russian). https:// doi.org/10.14498/vsgtu1711.

20. Ivanychev D. A. A boundary state method for solving a mixed problem of the theory of anisotropic elasticity with mass forces, Vestn. Tomsk. Gos. Univ. Mat. Mekh., 2021, no. 71, pp. 63-77 (In Russian). https://doi.org/10.17223/19988621/71/6.

21. Penkov V.B., Rybakova M. R., Satalkina L. V. Application of the Schwarz algorithm to spatial problems of elasticity theory, Vesti Vuzov Chernozemya, 2015, no. 2 (40), pp. 23-31 (In Russian). 